\title{
Rotation Profile of Kepler-63 from Planetary Transits
}

\author{
Yuri Netto and Adriana Valio \\ Centro de Rádio Astronomia e Astrofísica Mackenzie (CRAAM), São Paulo, Brasil \\ email: dirceuyuri@hotmail.com
}

\begin{abstract}
Currently it is possible to estimate the rotation profile of a star that harbours a planet in an orbit such that it eclipses the star periodically. During one of these transits, the planet may occult a spot on the photosphere of the star, causing small variations in its light curve. By detecting the same spot in a later transit, it is possible to estimate the stellar rotation period. Here we present the results of this model for the case of the star Kepler-63, which has a planet in an orbit with high obliquity. This means that the planetary eclipse occults many latitude bands of the star, from near the equator to the poles. The results show that Kepler-63 has differential rotation of $0.133 \mathrm{rd} / \mathrm{d}$ and a relative differential rotation of $11.4 \%$.
\end{abstract}

Keywords. stars: rotation - starspots - planetary system - star: individual: Kepler-63

\section{Introduction}

A stellar rotation profile can be estimated if the star is eclipsed periodically by an orbiting planet. During one of these transits, the planet may occult a spot on the photosphere of the star, causing small variations in the transit light curve. By monitoring the positions of these spots in subsequent transits, it is possible to estimate the rotation period of a star (Silva-Valio 2008). Currently there are a total of over 1900 confirmed planets, and more than 1200 planets eclipse their host stars. Some stars with planets detected by CoRoT and Kepler satellites have been analysed and their rotation profiles obtained by this method (Silva-Valio \& Lanza 2011).

\section{Starspot model applied to Kepler-63}

Kepler-63 is a young, solar-like star (age $\sim 210 \mathrm{Myr}$ ) (Sanchis-Ojeda et al. 2013) that displays high activity. It has mass of $0.984 M_{\odot}$ and radius of $0.901 R_{\odot}$. The planet Kepler-63b has approximately 0.4 Jupiter masses and an orbital period of 9.42 days. Interestingly, the planet presents a polar orbit (Sanchis-Ojeda et al. 2013). Figure 1 shows the configuration of the stellar inclination and the planet transit, according to SanchisOjeda et al. (2013). The average rotation period of the star is obtained by analysing the Lomb-Scargle periodogram, which shows a strong peak at 5.4 days.

We applied a model that simulates planetary transits in front of a star (Silva 2003). The model assumes a synthetized image of a limb-darkened star with a planet on a circular orbit. The spots cause a slight increase in the intensity during a few minutes of the transit. The fit of the light curve by this model allows us to determine the physical characteristics of the spots, such as size, temperature and location.

This model was applied to the 150 observed transits of Kepler-63, using the stellar parameters reported in Sanchis-Ojeda et al. (2013). Some values had to be refined, such as the scaled semi-major axis and planet-to-star radius ratio, to better fit the transit 


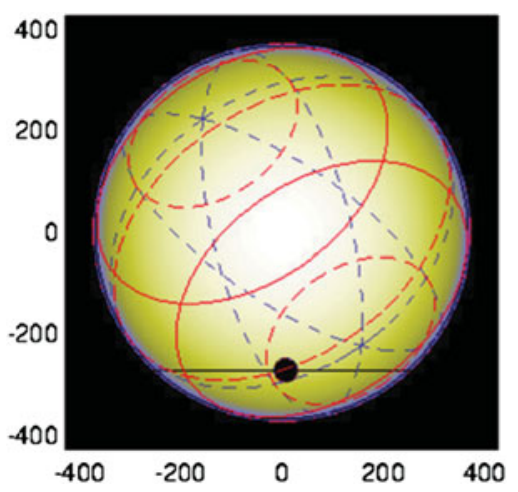

Figure 1. Planetary transit simulation for a planet on a polar orbit around Kepler-63.

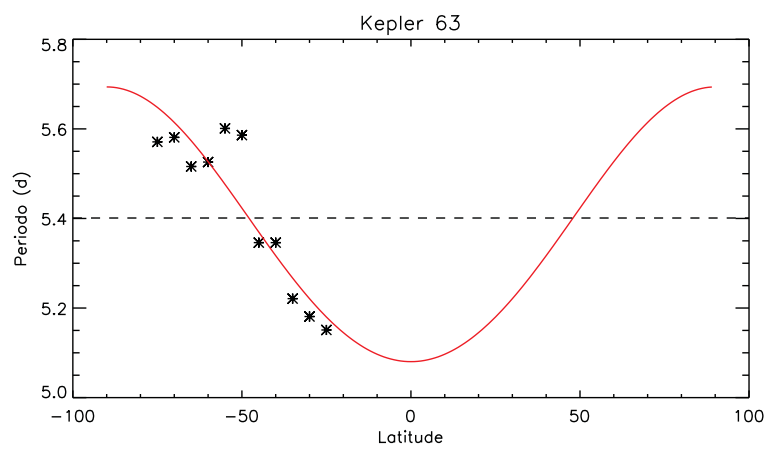

Figure 2. Kepler-63 rotation profile.

light curve. The results from the detection of 297 spots are listed in Table 1 in the form of mean values of the physical parameters.

\begin{tabular}{lcc|lcc} 
Parameters & Unit & Mean Value & Parameters & Unit & Mean Value \\
\hline Radius & $\left(R_{p}\right)$ & $0.8 \pm 0.3$ & Radius & $(\mathrm{km})$ & $(33 \pm 12) \cdot 10^{3}$ \\
Intensity & $\left(I_{c}\right)$ & $0.47 \pm 0.16$ & Temperature & $(\mathrm{K})$ & $4700 \pm 400$ \\
\hline
\end{tabular}

Table 1. Mean values of physical parameters of the star Kepler-63.

Due to the obliquity of the tilted stellar axis, the planet transit covers a range of stellar latitudes of Kepler-63 (Sanchis-Ojeda et al. 2013). To analyse the data obtained from the spots inferred by the light curve fits it was necessary to apply a rotation matrix to the position of the spots, since the model assumes orbits that are coplanar with the stellar equator. Thus, we obtained the spot position on the reference frame that rotates with the star.

The spots were separated into latitude bands of $5^{\circ}$. Using the model described in SilvaValio \& Lanza (2011) we determined the rotation period for each of these latitude bands. Assuming solar-like differential rotation $\left(\Omega=A-B \sin ^{2}(\right.$ lat $\left.)\right)$ and the set of rotation period values obtained for different latitudes yields the rotation profile of Kepler-63 presented in Figure 2. Through these values, it is possible to calculate the differential rotation, $\Delta \Omega$, and relative differential rotation, $\Delta \Omega / \Omega_{0}(\%)$. For Kepler-63 we obtained $\Delta \Omega / \Omega_{0}=11.4 \%$ and $\Delta \Omega=0.133 \mathrm{rd} / \mathrm{d}$. 


\section{Summary and Conclusions}

Assuming a differential rotation period like that of our Sun, we determined the differential rotation and relative differential rotation for Kepler-63.

The differential rotation of Sun $(\Delta \Omega=0.050 \mathrm{rd} / \mathrm{d})$ and differential rotation of CoRoT$2(\Delta \Omega=0.042 \mathrm{rd} / \mathrm{d})$, both stars of $\mathrm{G}$ spectral type (Valio 2013), present values more than twice lower than what we found for Kepler-63. Nevertheless, Kepler-63 presents a relative rotation period quite different from these stars. CoRoT-2, with an age between 0.13 and $0.5 \mathrm{Gyr}$, has a relative differential rotation of $\Delta \Omega / \Omega_{0}=3.0 \%$, whereas the much older Sun ( 4.6 Gyr) presents a value of $22.1 \%$ (Valio 2013), twice that of Kepler-63.

\section{References}

Sanchis-Ojeda, R., Winn, J. A., Marcy, et al. 2013, ApJ, 775, 54

Silva, A. V. R. 2003, ApJLett, 585, 147

Silva-Valio, A. 2008, ApJLett, 683, 179

Silva-Valio, A. \& Lanza, A. F. 2011, A\& $A$, 529, A36

Valio, A. 2013, New Quests in Stellar Astrophysics III: A Panchromatic View of Solar-Like Stars, With and Without Planets, 472, 239 